\title{
Integrative analysis of the heat shock response in Aspergillus fumigatus
}

Daniela Albrecht ${ }^{1}$, Reinhard Guthke ${ }^{1}$, Axel A Brakhage ${ }^{2,3}$, Olaf Kniemeyer ${ }^{2,3^{*}}$

\begin{abstract}
Background: Aspergillus fumigatus is a thermotolerant human-pathogenic mold and the most common cause of invasive aspergillosis (IA) in immunocompromised patients. Its predominance is based on several factors most of which are still unknown. The thermotolerance of A. fumigatus is one of the traits which have been assigned to pathogenicity. It allows the fungus to grow at temperatures up to and above that of a fevered human host. To elucidate the mechanisms of heat resistance, we analyzed the change of the A. fumigatus proteome during a temperature shift from $30^{\circ} \mathrm{C}$ to $48^{\circ} \mathrm{C}$ by $2 \mathrm{D}$-fluorescence difference gel electrophoresis (DIGE). To improve $2 \mathrm{D}$ gel image analysis results, protein spot quantitation was optimized by missing value imputation and normalization. Differentially regulated proteins were compared to previously published transcriptome data of $A$. fumigatus. The study was augmented by bioinformatical analysis of transcription factor binding sites (TFBSs) in the promoter region of genes whose corresponding proteins were differentially regulated upon heat shock.

Results: 91 differentially regulated protein spots, representing 64 different proteins, were identified by mass spectrometry (MS). They showed a continuous up-, down- or an oscillating regulation. Many of the identified proteins were involved in protein folding (chaperones), oxidative stress response, signal transduction, transcription, translation, carbohydrate and nitrogen metabolism. A correlation between alteration of transcript levels and corresponding proteins was detected for half of the differentially regulated proteins. Interestingly, some previously undescribed putative targets for the heat shock regulator Hsf1 were identified. This provides evidence for Hsf1dependent regulation of mannitol biosynthesis, translation, cytoskeletal dynamics and cell division in A. fumigatus. Furthermore, computational analysis of promoters revealed putative binding sites for an AP-2alpha-like transcription factor upstream of some heat shock induced genes. Until now, this factor has only been found in vertebrates.

Conclusions: Our newly established DIGE data analysis workflow yields improved data quality and is widely applicable for other DIGE datasets. Our findings suggest that the heat shock response in A. fumigatus differs from already well-studied yeasts and other filamentous fungi.
\end{abstract}

\section{Background}

Aspergillus fumigatus is a ubiquitous fungus that can be isolated from habitats such as soil or compost [1]. In the last decades, it has become the primary mold pathogen of humans. Especially in immunocompromised patients, the fungus is responsible for life-threatening infections. Such invasive aspergillosis (IA) is associated with a mortality rate of up to $90 \%$ [2]. The small size of spores permits ready access to the lung alveoli, which are the primary site of infection. A. fumigatus is highly

\footnotetext{
* Correspondence: olaf.kniemeyer@hki-jena.de

${ }^{2}$ Department of Molecular and Applied Microbiology, Leibniz Institute for Natural Product Research and Infection Biology, Hans-Knöll-Institute, Jena, Germany
}

(C) 2010 Albrecht et al; licensee BioMed Central Ltd. This is an Open Access article distributed under the terms of the Creative Commons Attribution License (http://creativecommons.org/licenses/by/2.0), which permits unrestricted use, distribution, and reproduction in any medium, provided the original work is properly cited.
列 $70^{\circ} \mathrm{C}$ which convey thermo- and stress resistance at high temperature may also contribute to the virulence of this mold [3]. However, only few genes have been shown to be involved in thermotolerance of A. fumigatus so far and no direct link to virulence was found yet. A strain lacking the $o$-mannosyltransferase gene Afpmt 1 was shown to be impaired in growth above $37^{\circ} \mathrm{C}$ [4] but did not show attenuation in virulence. A similar phenotype was shown for the thermotolerance protein ThtA, the function of which is unknown [5]. The nucleolar protein CgrA that is involved in ribosome biogenesis was shown to be essential for growth at $37^{\circ} \mathrm{C}$ in vitro and in infected mice, but was dispensable at $22^{\circ} \mathrm{C}$ [3]. 
Thermoresistance in A. fumigatus, therefore, is polygenic and mediated by numerous factors. For this reason, global studies are required to identify sets of transcripts or proteins, which are important for growth at elevated temperatures. Furthermore, a comparison of the heat shock response of A. fumigatus to that in other fungi may reveal differences that help to explain its thermotolerance.

The heat shock response of the budding yeast Saccharomyces cerevisiae is the best characterised both on the transcriptome and proteome level [6-12]. One of the primary effects of heat shock in this organism is unand refolding of misfolded proteins which is mediated by heat shock proteins (HSPs, chaperones). Moreover, the expression of genes or proteins involved in cell wall integrity, cytoskeleton organisation, small molecular and vesicular transport, energy generation, defense against oxidative stress, signal transduction, carbohydrate metabolism, ubiquitination, and proteolysis are induced.

The heat and general stress response in yeast is regulated by the transcription factors Hsf1, Msn2/Msn4 and Hac1. The transcriptional regulator Hsf1 binds to specific repeat sequences (nGAAn or nTTCn) termed heat shock elements (HSE). Upon heat shock, Hsf1 induces the expression of chaperones and many other genes (reviewed in $[13,14]$ ). The second regulatory system of the heat shock response consists of Msn2 and Msn4. They play central roles in response to several stresses by activating gene expression via the stress response element (STRE) (reviewed in $[13,7]$ ). The third component is Hac1. This transcription factor activates the expression of genes the products of which promote protein folding in the endoplasmic reticulum (ER) and degradation of incorrectly folded proteins (reviewed in [15]).

In A. fumigatus, the global regulatory network induced upon heat shock has so far only been investigated on the transcriptome level [16-18]. To gain further insight into the heat shock response of $A$. fumigatus at the level of the proteome, we applied 2D-fluorescence difference gel electrophoresis (DIGE). This technique is superior to classical gel-based proteomics approaches. It enables separating different samples labelled with spectrally resolvable fluorescent dyes on the same $2 \mathrm{D}$ gel. This reduces experimental gel-to-gel variation and provides increased statistical confidence. However, raw DIGE data still reflect technical and biological variation. Additionally, missing intensity values may be a problem, depending on the software used for gel image analysis [19]. It was shown that the normalization methods of commercial software are not able to eliminate all bias and produce different results depending on the image analysis software used [20,21]. Therefore, several attempts have been made to reduce noise and to improve the quality of datasets by various statistical methods [19-21].

Here, we characterized the protein expression of $A$. fumigatus following a temperature shift from $30^{\circ} \mathrm{C}$ to $48^{\circ} \mathrm{C}$. This rise in temperature induces a clear heat shock response in A. fumigatus and corresponds to temperature conditions in compost piles. Data analysis was optimized by imputing missing intensity values and subsequent data normalisation. Additionally, transcriptome and sequence information was used to achieve a comprehensive view of the A. fumigatus heat shock response. Furthermore, we compared our data with global studies of other fungi to elucidate possible factors which may distinguish a thermotolerant mold from mesophilic species. Our study revealed novel putative Hsf1-targets in A. fumigatus.

\section{Results}

\section{Experimental Design}

The DIGE technique has the advantage of increasing replicate number whilst keeping the number of $2 \mathrm{D}$ gels required relatively low. It allows multiplexing of up to three samples, labeled with spectrally resolvable fluorescent dyes (Cy3, Cy5, Cy2), in one gel [22]. The comigration of proteins in one gel eliminates running differences between those samples. The additional use of a pooled internal standard results in better inter- and intra-gel matching of spots. It has been shown that this technique is able to generate statistically significant data with fewer $2 \mathrm{D}$ gels than traditional $2 \mathrm{D}$ gel electrophoresis [23]. Furthermore, the application of CyDyes ensures a linear detection over a wide range of protein abundance with around four orders of magnitude $[24,25]$. Applying the reference design (Figure 1B), as most studies do, results in the use of half of the gels for measuring the reference. Loop design (Figure 1C), is more suitable, because it reduces cost and experimental effort and, at the same time, it produces statistically sound data [26]. Extended loop design (Figure 1D) has the additional advantage of high intersample connectivity.

In this study, reference and extended loop design were applied. Loop design resulted in less missing data than reference design ( $31 \%$ vs. $46 \%$ ). Therefore, more data could be used for further analyses (982 vs. 456 spots). This also resulted in the detection of more significant differentially regulated spots (183 vs. 55). Because of this, we focused on the data generated by application of the extended loop design.

\section{Image and data analysis}

Imputation methods were selected from a set of described approaches which have previously been applied to proteomic datasets (e.g., [19,27-29]). The quality of these methods was assessed by several tests. 


\section{$2 x$ : \\ 4 gels 0 \\ 4 gels 30 \\ 4 gels 60 \\ 4 gels 120 \\ 4 gels 180}

Total: 40 gels

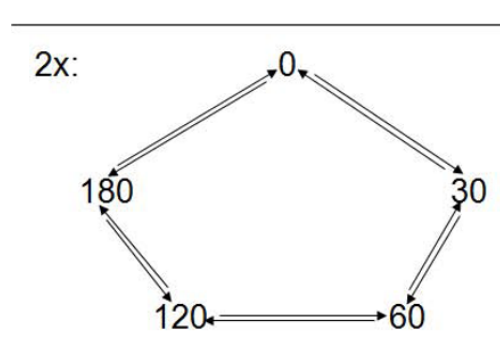

Total: 20 gels
$2 x$

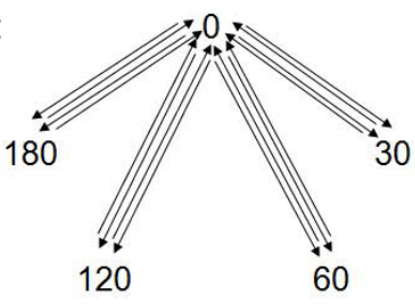

Total: 32 gels

B

$2 x$ :

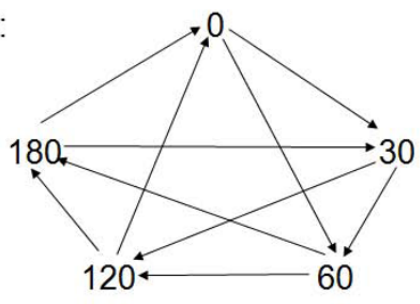

Total: 20 gels

Figure 1 Possible experimental designs for time series experiments. 0, 30, 60, 120, and 180 each represent one biological sample taken at this time point (min.) after temperature shift; arrows point from the Cy3 labeled sample to the Cy5 labeled sample and represent gels; A - non DIGE; B - DIGE, reference design; C - DIGE, loop design; D - DIGE, extended loop design.

The combination of minimal imputation and k-nearest neighbors was found to perform best [30]. It defines spots appearing in less than $25 \%$ of the gels of one experimental condition as noise. Present spot values from similarly regulated spots are used for imputing missing data of spots with less than $25 \%$ missing values across all gels. To our knowledge, the combination of these two methods was used and evaluated here for the first time and is also eligible for other DIGE studies.

The normalization procedure, using variance stabilization (vsn) first and local polynomial regression (loess) subsequently, was tested against no normalization, DeCyder standard normalization and normalization using only vsn or loess. The newly introduced combination of vsn and loess produced the best result in terms of noise reduction [31].

The whole data analysis workflow is publicly available as tool DIGE analyzer within the data warehouse OmniFung [32] at http://www.omnifung.hki-jena.de/Rpad/ DIGE_analyzer.Rpad. Also, all experimental data produced in this study are stored in this data warehouse and are available by public login.

\section{Differentially regulated proteins at high temperature}

Preliminary 2D-gel electrophoresis studies revealed only a small change in protein expression following an increase of the cultivation temperature from $30^{\circ} \mathrm{C}$ to $37^{\circ}$ C (data not shown). Concordantly, transcriptome analysis of temperature shift experiments revealed a higher expression of heat-shock responsive genes following a shift from $30^{\circ} \mathrm{C}$ to $48^{\circ} \mathrm{C}$ in comparison to $37^{\circ} \mathrm{C}$ [17]. We therefore decided to focus our protein expression studies on the heat shock response at $48^{\circ} \mathrm{C}$ (representing temperature in compost). This also allows a better comparison of data from less thermotolerant, mesophilic fungi, such as $S$. cerevisiae, where a temperature of $37^{\circ} \mathrm{C}$ already induces a clear heat shock response.

1886 spots were detected in the gels (Figure 2). A set of 183 spots was analyzed further. 91 of them, representing 64 different proteins, were identified by MS (additional file 1, MS_results.xls). The number of differently expressed proteins is similar to previous 2D-gel electrophoresis studies of the heat shock response in $S$. cerevisiae [10,33], Penicillium marneffei [34] and Aspergillus flavus [35]. All spots representing the same protein (up to five for some proteins) showed a very similar regulation. Therefore, the median value of these spots was calculated for each time point to define a protein as up- or downregulated. 54 proteins appeared to be upregulated, whereas 10 showed downregulation (Table 1 ). The putative functions identified for these proteins can be divided into several groups: many proteins have functions in protein folding (chaperones), in the organization of the cytoskeleton, transcription, translation and the oxidative stress response. The flavohemoprotein 


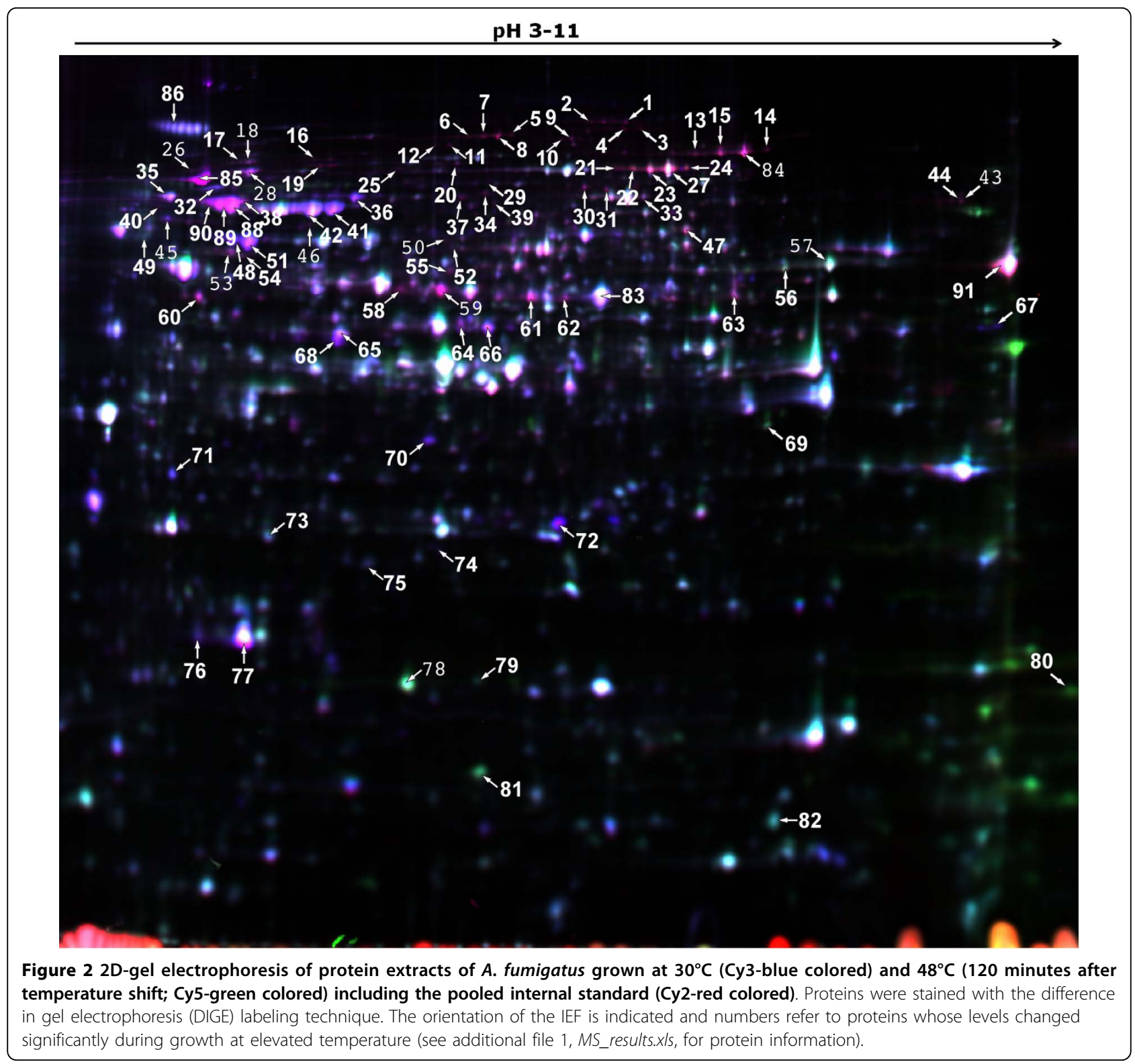

AFUA_4G03410 acts as an NO detoxification enzyme [36] and the nitroreductase family protein AFUA_5G09910 has a putative role in maintaining the cellular oxidative and/or nitrosative balance [37]. The regulatory subunit of the protein phosphatase $2 \mathrm{~A}$ is presumably involved in hyphal growth [38] and in the repression of RNA-polymerase III transcription of rRNA and tRNA [39]. In addition, a large group of differentially regulated proteins was involved in amino acid biosynthesis and in carbohydrate metabolism: enzymes of glycolysis (phosphoglycerate kinase PgkA, hexokinase Kxk), TCA cycle (alpha-ketoglutarate dehydrogenase complex subunit Kgd1), pentose phosphate pathway (transketolase TktA; 6-phosphogluconate dehydrogenase
Gnd1), mannose metabolism (mannitol-1-phosphare dehydrogenase), lipid biosynthesis (ATP citrate lyase subunit 1, acetyl coenzyme A synthetase FacA), and an NADP-dependent isocitrate dehydrogenase were affected by heat shock.

The 64 significant differentially regulated and identified proteins were grouped according to qualitative features of their time courses. In grouping, up- and downregulation was discriminated. Both groups of proteins were subdivided into oscillating and non-oscillating proteins. A protein was defined as being oscillating when the slope of its time course changed its algebraic sign twice, once at 30 or 60 minutes (first extremum) and once at 
Table 1 Differentially regulated proteins in $A$. fumigatus after a temperature shift from $30^{\circ} \mathrm{C}$ to $48^{\circ} \mathrm{C}$.

\begin{tabular}{|c|c|c|c|c|c|}
\hline Protein & $T=30$ & $T=60$ & $T=120$ & $T=180$ & Osc \\
\hline \multicolumn{6}{|l|}{ Up regulated } \\
\hline \multicolumn{6}{|l|}{ Chaperone } \\
\hline AFUA_1G07440 (molecular chaperone Hsp70) & 1.95 & 2.45 & 2.06 & 2.45 & No \\
\hline AFUA_1G11180 (heat shock protein/chaperonin HSP78) & 1.18 & 1.24 & 0.77 & 1.33 & No \\
\hline AFUA_1G12610 (HSP 70 chaperone HSP88) & 1.19 & 1.06 & 0.59 & 1.34 & Yes \\
\hline AFUA_2G04620 (Hsp70 chaperone BiP/Kar2) & 0.22 & 0.84 & 1.50 & 1.68 & No \\
\hline AFUA_2G09290 (antigenic mitochondrial protein HSP60) & 0.43 & 1.28 & 2.17 & 1.88 & No \\
\hline AFUA_2G09960 (mitochondrial Hsp70 chaperone (Ssc70)) & 0.18 & 0.81 & 1.47 & 1.23 & No \\
\hline AFUA_3G14540 (heat shock protein Hsp30/Hsp42) & -0.24 & 1.23 & 2.37 & 2.35 & No \\
\hline AFUA_4G10010 (Hsp90 co-chaperone Cdc37) & 1.13 & 1.22 & 1.84 & 1.97 & No \\
\hline AFUA_4G12850 (calnexin) & 0.19 & 0.59 & 1.84 & 1.58 & No \\
\hline AFUA_5G04170 (molecular chaperone and allergen Mod-E/Hsp90/Hsp1) & 3.11 & 3.68 & 3.13 & 3.76 & No \\
\hline AFUA_5G07340 (DnaJ domain protein Psi) & 0.50 & 1.01 & 2.31 & 1.58 & No \\
\hline AFUA_7G01860 (heat shock protein (Sti1)) & 0.38 & 1.30 & 1.92 & 1.70 & No \\
\hline \multicolumn{6}{|l|}{ Cell wall and cytoskeleton } \\
\hline AFUA_1G02550 (tubulin alpha-1 subunit) & 1.04 & 0.92 & -0.19 & 0.48 & Yes \\
\hline AFUA_2G07420 (actin-bundling protein Sac6) & 1.48 & 1.27 & 0.30 & 1.16 & Yes \\
\hline \multicolumn{6}{|l|}{ Transport } \\
\hline AFUA_6G07120 (nuclear movement protein NudC) & -0.64 & 0.60 & 1.79 & 1.18 & Yes \\
\hline \multicolumn{6}{|l|}{ Energy generation } \\
\hline AFUA_2G13240 (V-type ATPase, B subunit) & 0.95 & 0.66 & 0.56 & 1.02 & No \\
\hline \multicolumn{6}{|l|}{ Defence against oxidative and nitrosative stress } \\
\hline AFUA_4G03410 (flavohemoprotein) & 0.73 & 0.90 & 0.97 & 1.04 & No \\
\hline AFUA_4G09110 (cytochrome c peroxidase Ccp1) & 0.00 & 1.29 & 1.92 & 2.03 & No \\
\hline AFUA_5G09910 (nitroreductase family protein) & -1.18 & -0.53 & 1.02 & 1.32 & No \\
\hline AFUA_6G02280 (allergen Asp F3) & -0.54 & 0.35 & 1.62 & 1.13 & Yes \\
\hline \multicolumn{6}{|l|}{ Signal transduction } \\
\hline AFUA_1G05610 (protein phosphatase 2a 65 kd regulatory subunit) & 1.47 & 1.47 & 0.98 & 1.27 & No \\
\hline AFUA_4G12450 (conserved lysine-rich protein) & -0.01 & 0.21 & 1.34 & 1.09 & No \\
\hline \multicolumn{6}{|l|}{ Carbohydrate metabolism } \\
\hline AFUA_1G10350 (phosphoglycerate kinase PgkA) & 0.12 & 0.42 & 1.08 & 0.80 & No \\
\hline AFUA_1G13500 (transketolase TktA) & 1.07 & 0.54 & -0.62 & -0.01 & Yes \\
\hline AFUA_2G05910 (hexokinase Kxk) & 1.39 & 1.27 & 0.35 & 0.73 & Yes \\
\hline AFUA_2G10660 (mannitol-1-phosphate dehydrogenase) & 1.70 & 1.67 & 1.09 & 1.46 & No \\
\hline AFUA_3G08660 (isocitrate dehydrogenase Idp1) & 1.19 & 0.95 & 0.08 & 0.42 & Yes \\
\hline AFUA_4G04680 (FGGY-family carbohydrate kinase) & 1.12 & 0.88 & 0.29 & 0.79 & Yes \\
\hline AFUA_4G11080 (acetyl-coenzyme A synthetase FacA) & 1.64 & 1.27 & -0.10 & 0.88 & Yes \\
\hline $\begin{array}{l}\text { AFUA_4G11650 (alpha-ketoglutarate dehydrogenase complex subunit } \\
\text { Kgd1) }\end{array}$ & 1.72 & 1.39 & 0.49 & 0.95 & Yes \\
\hline AFUA_6G04210 (mannosyl-oligosaccharide glucosidase) & 1.29 & 1.21 & 0.77 & 0.97 & No \\
\hline AFUA_6G04920 (NAD-dependent formate dehydrogenase AciA/Fdh) & 1.17 & 0.66 & -0.67 & 0.05 & Yes \\
\hline AFUA_6G08050 (6-phosphogluconate dehydrogenase Gnd1) & 2.21 & 1.85 & 0.55 & 1.03 & Yes \\
\hline AFUA_6G10650 (ATP citrate lyase, subunit 1) & 1.38 & 0.92 & -0.16 & 0.33 & Yes \\
\hline \multicolumn{6}{|l|}{ Nitrogen metabolism } \\
\hline AFUA_1G10130 (adenosylhomocysteinase) & 1.88 & 1.46 & 0.05 & 0.60 & Yes \\
\hline AFUA_1G12840 (nitrite reductase NiiA) & 2.75 & 2.30 & 1.19 & 1.54 & Yes \\
\hline AFUA_2G11260 (3-isopropylmalate dehydratase) & 1.18 & 0.90 & -0.09 & 0.44 & Yes \\
\hline AFUA_4G07360 (cobalamin-independent methionine synthase MetH/D) & 1.53 & 0.66 & -1.08 & -0.08 & Yes \\
\hline AFUA_4G07710 (pyruvate carboxylase) & 2.29 & 1.84 & 0.78 & 1.25 & Yes \\
\hline AFUA_4G10460 (homocitrate synthase) & 1.56 & 1.04 & 0.31 & 0.88 & Yes \\
\hline AFUA_4G13120 (glutamine synthetase) & 0.67 & 1.48 & 1.72 & 1.84 & No \\
\hline
\end{tabular}


Table 1: Differentially regulated proteins in A. fumigatus after a temperature shift from $30^{\circ} \mathrm{C}$ to $48^{\circ} \mathrm{C}$. (Continued)

\begin{tabular}{|c|c|c|c|c|c|}
\hline \multicolumn{6}{|l|}{ Ubiquitination and proteolysis } \\
\hline AFUA_4G09030 (aminopeptidase) & 1.58 & 1.31 & 0.51 & 1.30 & Yes \\
\hline AFUA_5G04330 (aminopeptidase) & 1.07 & 1.08 & 0.07 & 0.35 & Yes \\
\hline \multicolumn{6}{|l|}{ Protein biosynthesis/Translation } \\
\hline AFUA_1G02030 (eukaryotic translation initiation factor 3 subunit EifCb) & 1.33 & 0.64 & -0.10 & 0.43 & Yes \\
\hline AFUA_1G06390 (translation elongation factor EF-1 alpha subunit) & 2.00 & 1.28 & -0.51 & 0.69 & Yes \\
\hline AFUA_2G13530 (translation elongation factor EF-2 subunit) & 1.88 & 1.48 & -0.43 & 0.62 & Yes \\
\hline AFUA_3G08160 (eukaryotic translation initiation factor 4) & 1.81 & 1.48 & 0.84 & 1.18 & Yes \\
\hline AFUA_4G12920 (histidyl-tRNA synthetase, mitochondrial precursor) & 1.10 & 1.20 & 0.64 & 1.18 & Yes \\
\hline AFUA_5G05920 (glycyl-tRNA synthetase) & 1.56 & 1.26 & 0.23 & 0.83 & Yes \\
\hline AFUA_6G04570 (translation elongation factor eEF-1 subunit gamma) & 1.20 & 1.10 & 0.22 & 0.71 & Yes \\
\hline AFUA_7G05660 (translation elongation factor eEF-3) & 2.02 & 1.43 & -0.19 & 0.70 & Yes \\
\hline AFUA_8G03880 (alanyl-tRNA synthetase) & 1.37 & 0.80 & -0.22 & 0.32 & Yes \\
\hline \multicolumn{6}{|l|}{ Transcription } \\
\hline AFUA_1G15620 (DEAD box RNA helicase HelA) & 1.43 & 1.40 & 0.24 & 0.70 & Yes \\
\hline \multicolumn{6}{|l|}{ Cell cycle } \\
\hline AFUA_2G17110 (cell division control protein Cdc48) & 1.01 & 1.11 & 0.50 & 0.94 & Yes \\
\hline \multicolumn{6}{|l|}{ Down regulated } \\
\hline \multicolumn{6}{|l|}{ Chaperone } \\
\hline AFUA_2G13040 (mitochondrial co-chaperone GrpE) & -1.12 & -0.73 & 0.21 & -0.57 & Yes \\
\hline \multicolumn{6}{|l|}{ Transport } \\
\hline AFUA_5G03690 (CRAL/TRIO domain protein) & -1.29 & -0.61 & 0.37 & -0.53 & Yes \\
\hline \multicolumn{6}{|l|}{ Energy generation } \\
\hline AFUA_2G13010 (cytochrome c oxidase polypeptide vib) & -1.79 & -1.54 & -0.12 & -1.12 & Yes \\
\hline \multicolumn{6}{|l|}{ Carbohydrate metabolism } \\
\hline AFUA_1G09930 (glycerol dehydrogenase Gcy1) & -1.16 & -1.44 & -1.25 & -0.98 & No \\
\hline AFUA_3G09230 (carboxylesterase) & 0.14 & -0.69 & -1.07 & -1.07 & No \\
\hline \multicolumn{6}{|l|}{ Nitrogen metabolism } \\
\hline AFUA_3G09320 (serine hydroxymethyltransferase) & 0.06 & -0.67 & -1.01 & -1.12 & No \\
\hline \multicolumn{6}{|l|}{ Protein biosynthesis/Translation } \\
\hline $\begin{array}{l}\text { AFUA_6G02750 (nascent polypeptide-associated complex (NAC) } \\
\text { subunit) }\end{array}$ & -1.35 & -1.21 & -0.85 & -1.36 & No \\
\hline AFUA_6G12660 (40S ribosomal protein S10b) & -1.35 & -1.21 & -0.85 & -1.36 & No \\
\hline \multicolumn{6}{|l|}{ Transcription } \\
\hline AFUA_3G08580 (glycine-rich RNA-binding protein) & -1.27 & -1.18 & -0.84 & -2.09 & No \\
\hline AFUA_8G05300 (RNA polymerase II subunit 7) & -1.38 & -0.97 & -0.72 & -1.81 & No \\
\hline
\end{tabular}

Relative protein abundance is depicted as $\log _{2}$-ratio. Logarithmized normalized intensities of the respective time points were subtracted by logarithmized normalized intensity of time point $\mathrm{t}=0$. Osc describes whether the protein is oscillatingly regulated.

120 minutes (second extremum). Additionally, the difference between the $\log _{2}$-ratios of a spot at these time points had to be larger than the standard deviation of the $\log _{2}$-ratios from the whole time course of this spot (Figure 3). The grouping is in agreement with clusters calculated by fuzzy c-means algorithm $(\mathrm{fcm})$ for the corresponding transcriptomic data [16]. Chaperones were mostly non-oscillating upregulated, as were proteins involved in defense against oxidative stress and signal transduction. Also, most of the downregulated proteins were non-oscillating. Proteins of the metabolism as well as of transcription and translation were upregulated in an oscillating manner.
In addition to the manual annotation of the differentially regulated proteins with a functional class as described above, FunCat categorization [40] was conducted. Most affected proteins have binding functions or need cofactors and are related to metabolism, cell rescue, defence and virulence or protein fate. Since the categories on the top level of FunCat are quite broad, an enrichment analysis was conducted on the second level. The following categories displayed a p-value below 0.05 (in descending order of significance): protein folding and stabilization, nucleotide binding, stress response, protein binding, animal cell type differentiation, translation, cell death, phosphate metabolism, assembly of protein complexes, glycolysis and gluconeogenesis, fungal/ 

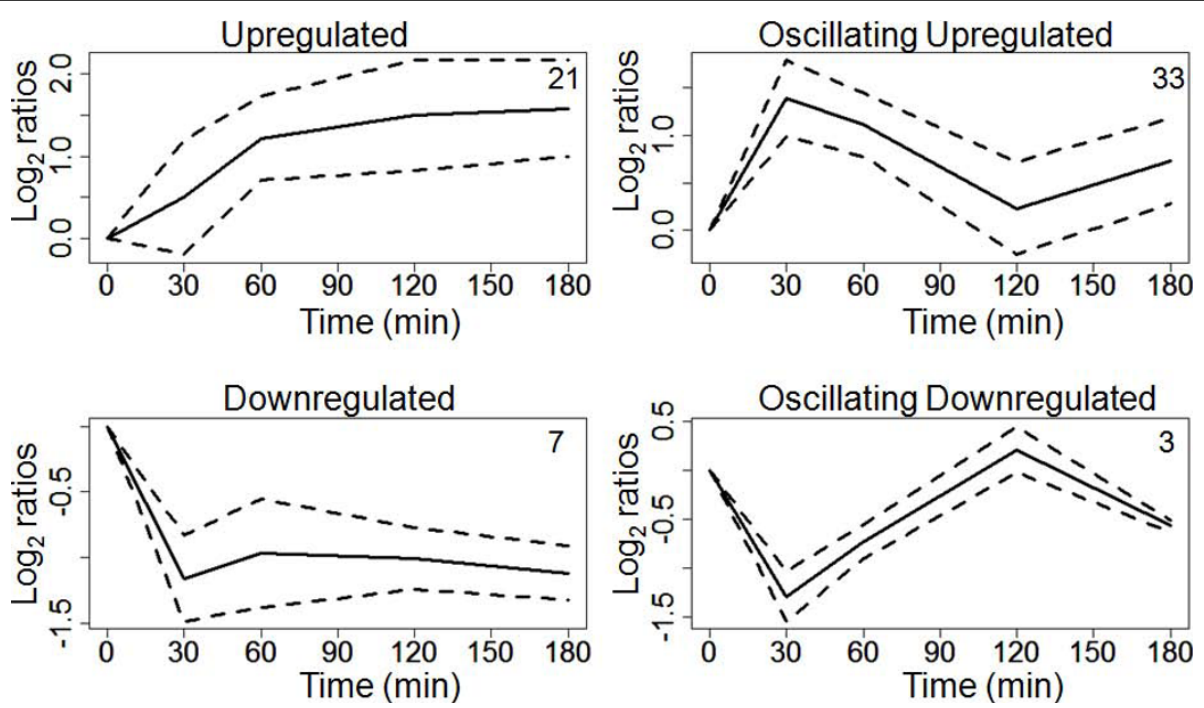

Figure 3 Differentially regulated proteins in the heat response form four groups with similar time courses. Solid lines represent median time courses and dashed lines the median absolute deviation of every time course group. Time is given in minutes and the relative abundance of protein spots in $\log _{2}$ ratios. In the right upper corner of every plot, the number of proteins in the group is displayed.

microorganismic development, tricarboxylic-acid pathway (citrate cycle, Krebs cycle, TCA cycle), aminoacyltRNA synthetases, cellular sensing and response, complex cofactor/cosubstrate binding, protein targeting, sorting and translocation, nucleus, and anaplerotic reactions. This enrichment shows that although many proteins belonged to the category metabolism, nonmetabolic categories are more important in the process of heat shock. FunCat categories of different hierarchical levels for all differentially regulated proteins as well as all p-values of the enrichment test at level two can be found in additional file 2 (funcat.xls).

\section{Relation of transcriptome and proteome data}

Whole genome analysis of only transcriptome or proteome data remains a reductionist approach, since both levels are strongly intertwined. To achieve a more holistic view, we analyzed previously published transcriptomic information. For half of the significant differentially regulated proteins the respective transcript was also found to be significantly regulated.

Low correlation of transcripts and corresponding proteins has been reported $[41,42]$. In this study, linear relationships were examined by Pearson correlation (PC) and non-linear relationships by Spearman correlation (SC). Median correlation for the whole time series of the 32 transcripts and respective proteins was -0.35 (PC) and -0.4 (SC). These values resulted from the fact that the expression of many proteins correlated negatively with their corresponding transcripts (see additional file 3, time_series_tr_pr.doc, for time course plots of transcripts and corresponding protein spots). PC ranged from -0.95 to 0.93 , SC from -0.94 to 0.94 . Both correlations depicted 22 of the 32 pairs of protein spots and transcripts showing negative values. Only 5 (PC, 4 $\mathrm{SC})$ pairs showed good correlation $(>0.5)$. This means that most proteins seemed to be upregulated while their respective transcript was downregulated and vice versa. Since negative correlation between so many transcripts and their corresponding proteins is counterintuitive, a model of time delay was introduced. A protein is most probably not regulated at exactly the same time point as its transcript due to the fact that translation follows transcription. Therefore, the contradictory behavior of a protein in respect to its transcript can be the result of earlier events, before heat shock was applied. By analyzing time-shifted correlation between transcriptomic and respective proteomic data, it was found that different proteins showed different time delays (Table 2). A maximum delay of 105 minutes was allowed. Using this approach, all PC between a transcript and its respective protein became greater than 0.51 and the median correlation was good with a value of 0.84 . Median correlation of SC was 0.89. Four SCs were below 0.5 (one remained below 0). For most proteins, the delay with the best correlation was the same for PC and SC. However, for eight proteins, there were differences of 15 minutes. This indicates that linear and non-linear dependencies between transcriptomic and proteomic data play a role. For one protein, a large difference of 105 minutes was calculated. This protein was the only one, where SC was below 0 . Taken together, the following picture appeared: eight proteins responded in the first 30 minutes after transcript regulation (no or short delay). 18 proteins were 60 to 90 minutes delayed (medium delay), and five 
Table 2 Comparison of the regulation on protein and transcript level.

\begin{tabular}{|c|c|c|}
\hline Protein & $\begin{array}{c}\text { PC of protein to transcript } \\
\text { (no delay/best delay } \\
\text { (best delay in minutes)) }\end{array}$ & $\begin{array}{c}\text { SC of protein to transcript } \\
\text { (no delay/best delay } \\
\text { (best delay in minutes)) }\end{array}$ \\
\hline \multicolumn{3}{|l|}{ Chaperone } \\
\hline AFUA_1G07440 (molecular chaperone Hsp70) & $0.48 / 0.52(15)$ & $0.26 / 0.52(15)$ \\
\hline AFUA_1G11180 (heat shock protein/chaperonin HSP78) & $0.44 / 0.52(15)$ & $0.31 / 0.52(15)$ \\
\hline AFUA_1G12610 (HSP 70 chaperone HSP88) & $0.72 / 0.72(0)$ & $0.60 / 0.60(0)$ \\
\hline AFUA_2G09290 (antigenic mitochondrial protein HSP60) & $0.33 / 0.86(75)$ & $0.09 / 0.86(75)$ \\
\hline AFUA_2G13040 (mitochondrial co-chaperone GrpE) & $0.80 / 0.80(0)$ & $0.83 / 0.83(0)$ \\
\hline AFUA_3G14540 (heat shock protein Hsp30/Hsp42) & $-0.36 / 0.95(105)$ & $-0.51 / 0.89(90)$ \\
\hline AFUA_4G10010 (Hsp90 co-chaperone Cdc37) & $0.18 / 0.76(105)$ & $-0.03 /-0.03(0)$ \\
\hline AFUA_5G04170 (molecular chaperone and allergen Mod-E/Hsp90/Hsp1) & $0.60 / 0.75(15)$ & $0.14 / 0.58(30)$ \\
\hline AFUA_5G07340 (DnaJ domain protein Psi) & $-0.35 / 0.82(75)$ & $-0.09 / 0.96(90)$ \\
\hline AFUA_7G01860 (heat shock protein (Sti1)) & $0.02 / 0.9(90)$ & $-0.09 / 0.96(90)$ \\
\hline \multicolumn{3}{|l|}{ Cell wall and cytoskeleton } \\
\hline AFUA_1G02550 (tubulin alpha-1 subunit) & $-0.52 / 0.97(60)$ & $-0.43 / 0.98(60)$ \\
\hline \multicolumn{3}{|l|}{ Transport } \\
\hline AFUA_5G03690 (CRAL/TRIO domain protein) & $-0.73 / 0.78(60)$ & $-0.77 / 0.88(75)$ \\
\hline AFUA_6G07120 (nuclear movement protein NudC) & $-0.24 / 0.68(90)$ & $-0.60 / 0.86(90)$ \\
\hline \multicolumn{3}{|l|}{ Defence against oxidative and nitrosative stress } \\
\hline AFUA_4G09110 (cytochrome c peroxidase Ccp1) & $0.76 / 0.94(105)$ & $0.60 / 0.94(105)$ \\
\hline AFUA_5G09910 (nitroreductase family protein) & $-0.75 / 0.83(105)$ & $-0.71 / 0.43(105)$ \\
\hline \multicolumn{3}{|l|}{ Signal transduction } \\
\hline AFUA_1G05610 (protein phosphotase 2a 65 kd regulatory subunit) & $-0.60 / 0.88(90)$ & $-0.54 / 0.96(90)$ \\
\hline \multicolumn{3}{|l|}{ Carbohydrate metabolism } \\
\hline AFUA_2G05910 (hexokinase Kxk) & $-0.95 / 0.91(90)$ & $-0.83 / 0.96(90)$ \\
\hline AFUA_2G10660 (mannitol-1-phosphate dehydrogenase) & $-0.13 / 0.51(15)$ & $0.03 / 0.57(15)$ \\
\hline AFUA_3G08660 (isocitrate dehydrogenase Idp1) & $-0.76 / 0.91(90)$ & $-0.77 / 0.93(90)$ \\
\hline AFUA_4G11080 (acetyl-coenzyme A synthetase FacA) & $-0.79 / 0.97(90)$ & $-0.71 / 0.93(90)$ \\
\hline AFUA_6G10650 (ATP citrate lyase, subunit 1) & $-0.450 / 0.94(60)$ & $-0.54 / 0.97(60)$ \\
\hline \multicolumn{3}{|l|}{ Nitrogen metabolism } \\
\hline AFUA_1G12840 (nitrite reductase NiiA) & $-0.22 / 0.95(30)$ & $-0.20 / 0.83(30)$ \\
\hline AFUA_2G11260 (3-isopropylmalate dehydratase) & $-0.34 / 0.93(105)$ & $-0.37 / 0.94(105)$ \\
\hline AFUA_2G13530 (bifunctional tryptophan synthase TRPB) & $-0.85 / 0.85(90)$ & $-0.83 / 0.93(75)$ \\
\hline AFUA_4G07360 (cobalamin-independent methionine synthase MetH/D) & $-0.83 / 0.81(75)$ & $-0.83 / 0.96(90)$ \\
\hline AFUA_4G07710 (pyruvate carboxylase) & $-0.54 / 0.96(90)$ & $-0.49 / 0.93(90)$ \\
\hline AFUA_4G10460 (homocitrate synthase) & $-0.73 / 0.86(75)$ & $-0.60 / 0.89(90)$ \\
\hline \multicolumn{3}{|l|}{ Protein biosynthesis/Translation } \\
\hline AFUA_1G02030 (eukaryotic translation initiation factor 3 subunit EifCb) & $-0.86 / 0.89(75)$ & $-0.89 / 0.88(75)$ \\
\hline AFUA_6G12660 (40S ribosomal protein S10b) & $-0.23 / 0.93(90)$ & $0.09 / 0.86(90)$ \\
\hline AFUA_7G05660 (translation elongation factor eEF-3) & $-0.17 / 0.70(105)$ & $-0.03 / 0.60(105)$ \\
\hline \multicolumn{3}{|l|}{ Transcription } \\
\hline AFUA_1G15620 (DEAD box RNA helicase HelA) & $-0.83 / 0.82(75)$ & $-0.94 / 0.86(90)$ \\
\hline AFUA_3G08580 (glycine-rich RNA-binding protein) & 0.93/0.93 (0) & $0.94 / 0.94(0)$ \\
\hline
\end{tabular}

If a protein was present in more than one spot, median correlation and mutual information of all spots is given. $\mathrm{PC}=\mathrm{Pearson}$ Correlation, $\mathrm{SC}=\mathrm{Spearman}$ Correlation. 
proteins responded 105 minutes after their transcript (strong delay). Proteins related to cell wall/cytoskeleton organization (tubulin- $\alpha$ Sac6), transport (NudC) and signal transduction (protein phosphatase 2a, conserved lysine rich protein) all responded with medium delay in respect to their transcript. Chaperones showed all different delays. Defense proteins against oxidative stress (allergen Asp F3, Ccp1) showed high delay. Carbohydrate metabolism (e.g., pentose phosphate shunt) and transcription proteins (RNA helicase HelA) responded with few or medium delay, while nitrogen metabolism and translation proteins responded with medium or strong delay.

\section{Comparison to yeast}

In yeast, heat shock is largely governed by the transcription factors Hsf1, Msn2/4 and Hac1. Many targets of those have already been elucidated $[11,12,14,43,44]$. Hsf1 was very recently found to be upregulated in $A$. fumigatus under heat shock [18]. We looked for putative
Hsf1 binding signatures $[12,14]$ in the genome of $A$. fumigatus. By using ScanProsite [45], 17 genes with a potential heat shock element (HSE) in their promoter region were detected (see Figure 4 for motif logos and Figure 5 for heatmap of transcript and protein regulation). Proteins probably regulated by Hsf1 include chaperones (HSP70, HSP78, mitochondrial HSP60, Sti1), enzymes of the oxidative stress response (cytochrome $\mathrm{C}$ peroxidase Ccp1, allergen Asp F3), signal transduction (protein phosphotase 2a 65 kd regulatory subunit, conserved lysine rich protein), carbohydrate and nitrogen metabolism (Hexokinase Kxk, mannitol-1-phosphate dehydrogenase, 6-phosphogluconate dehydrogenase Gnd1, nitrite reductase NiiA, 3-isopropylmalate dehydrogenase), protein biosynthesis/translation (eukaryotic translation initiation factor 4, histidyl-tRNA synthetase, DNAJ domain protein Psi) and transcription (glycinerich RNA-binding protein) (for explicit binding sites see additional file 4, sequence_analysis.xls). By using MEME [46], one additional chaperone (BiP/Kar2) and one
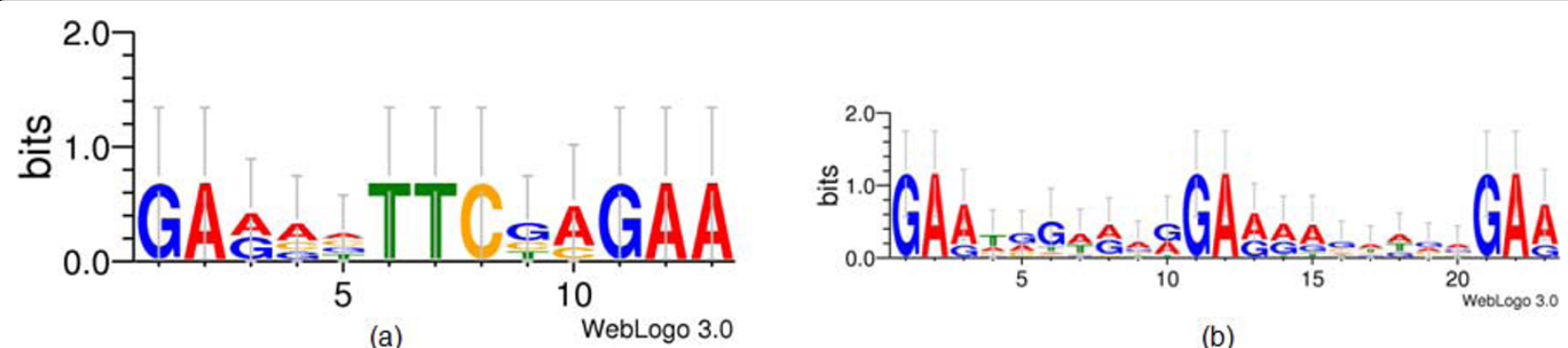

(b)
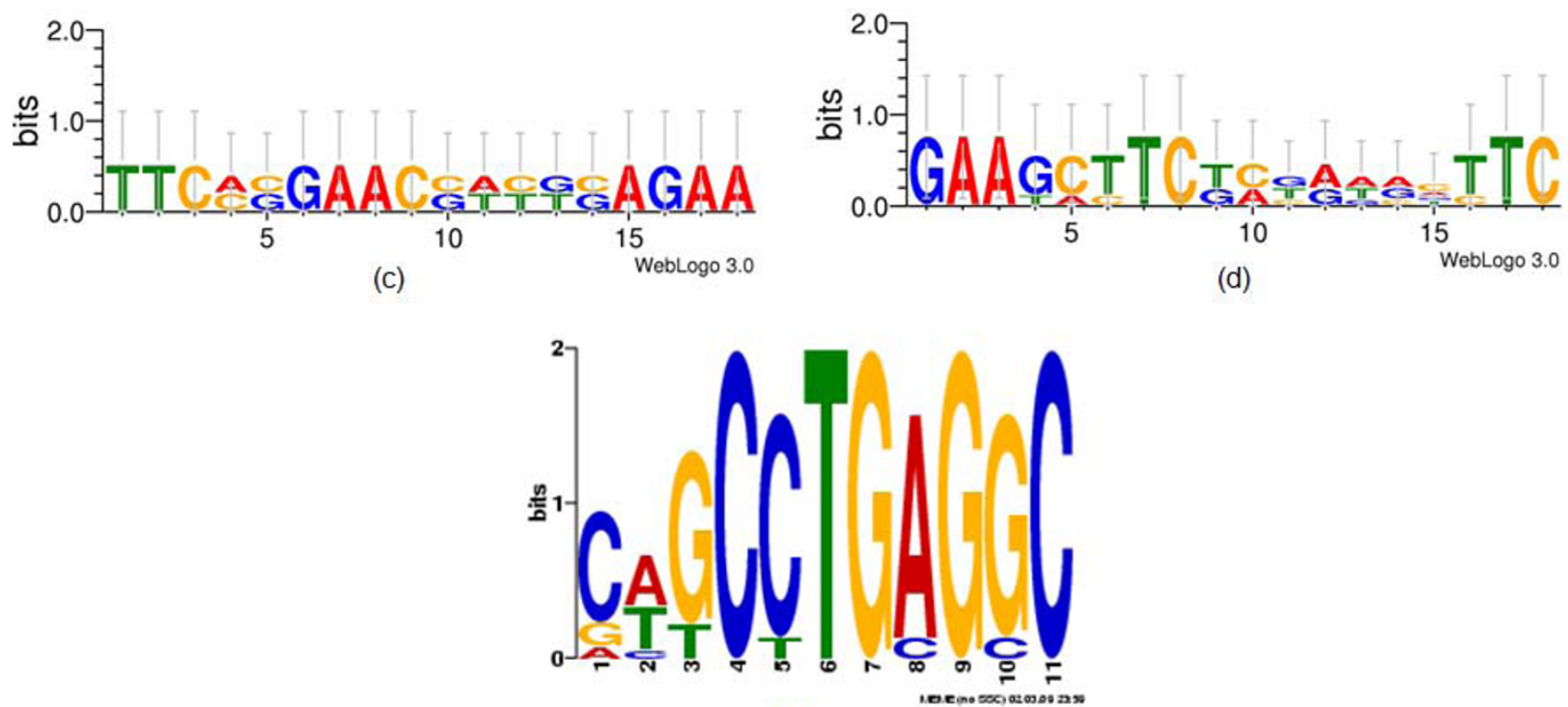

(e)

Figure 4 Sequence logos for Hsf1 and AP-2alphaA binding motifs as found in the promoter region of genes whose proteins were differentially regulated upon heat shock. Hsf: a (perfect), b (step), c (gap variant 1), d (gap variant 2), created with Weblogo, http://weblogo. threeplusone.com/; AP-2alphaA: e, created with MEME. 


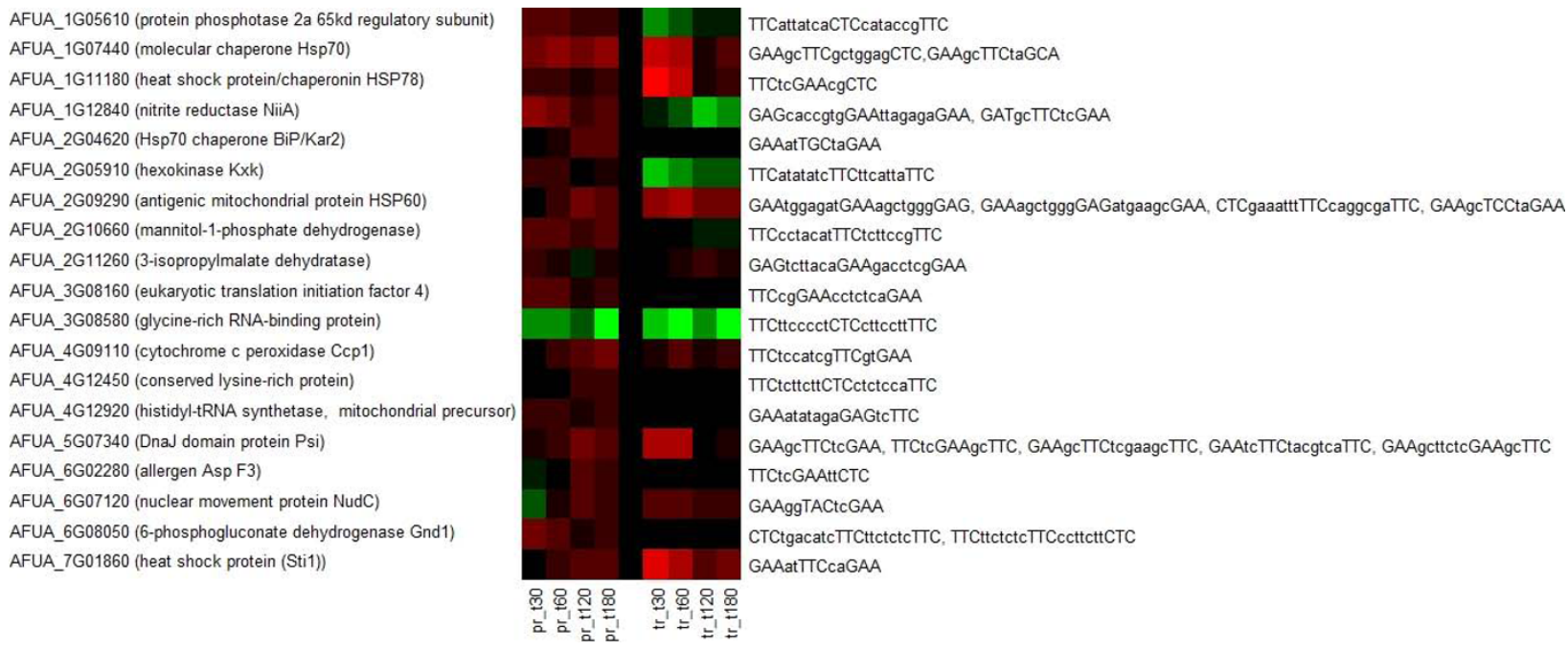

TाCattatcaCTC $\operatorname{cataccg} T \mathrm{C}$

GAAgcTTCgctggagCTC,GAAgcTTCtaGCA

TTCtcGAAcgCTC

GAGcaccgtgGAAttagagaGAA, GATgcTTCtcGAA

GAAatTGCTaGAA

TTCatatatcTTCttcattaTTC

GAAtggagatGAAagctgggGAG, GAAagctgggGAGatgaagcGAA, CTCgaaatttTCcaggcgaTTC, GAAgcTCCtaGAA TTCcctacatTTCtcttccgTTC

GAGtcttacaGAAgacctcgGAA

TTCcgGAAcctctcaGAA

TTCttcccctCTCcttccttTTC

TTCtccatcgTTCgtGAA

TTCtcttcttCTCctctccaTTC

GAAatatagaGAGtcTTC

GAAgc TTCtcGAA, TTCtcGAAgcTTC, GAAgcTCtcgaagc TTC, GAAtcTTCtacgtcaTTC, GAAgcttctcGAAgc $T C$ TTCtCGAAttCTC

GAAggTACtcGAA

CTCtgacatcTTCttctctcTTC, TTCttctctcTTCccttcttCTC

GAAatTCCaGAA

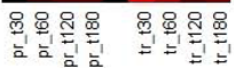

Figure 5 Heatmap of potentially Hsf1 regulated proteins and transcripts. Red color depicts upregulation, green color depicts downregulation. The gene/protein names are shown as well as the detected HSE.

transport protein (nuclear movement protein NudC) with slightly modified HSE motifs were found. Transcriptional activation of genes coding for chaperones is well known from yeast and higher organisms, but for some other genes (Ccp1, protein phosphotase 2a, conserves lysine rich protein, mannitol-1-phosphate dehydrogenase, Idp1, 3-isopropylmalae dehydrogenase, eukaryotic translation initiation factor 4, histidyl-tRNA synthetase, glycine-rich RNA-binding protein, NudC) regulation by Hsf1 has not been previously elucidated for yeast (for comparison with yeast homologues see additional file 4). For the transcription factors Msn2/4 and Hac1 neither ScanProsite nor MEME provided useful results, since the binding motifs are very short. In addition to the Hsf1 binding sites, MEME identified a possible binding motif for an AP-2alphaA-like transcription factor in 11 of the 64 differentially regulated proteins (AFUA_1G05610, AFUA_4G07710, AFUA_1G10130, AFUA_5G04170, AFUA_5G07340, AFUA_2G17110, AFUA_2G10660, AFUA_3G09320, AFUA_6G12660, AFUA_1G02030, AFUA_7G01860; see Figure 4 for motif logo). The AP-2 family of transcription factors regulates proliferation and differentiation during embryonic development in animals [47]. To date, no AP-2 homologues have been detected in any eukaryotic microorganism.

\section{Discussion}

Proteome analysis based on the DIGE technique was used to explore how protein expression changes over time in response to a temperature shift from $30^{\circ} \mathrm{C}$ to $48^{\circ}$ C. Data pre-processing methods (missing value imputation, normalization, filtering) were assessed and applied to improve the outcome of the image analysis.

The imputation and normalization approach used in this study combined previously described methods. Although each single method has been described before $[21,28,48,49]$, the combination of them has not been used and validated for proteomics data so far. These methods can in principle be applied to any DIGE dataset by using the online tool DIGE analyzer.

Grouping of significant differentially regulated proteins resulted in two groups with straight up- or downregulation and two groups that depicted oscillatory behavior. This can be explained by feedback loops in the process of heat shock response. Since the absolute value of the second extremum was in both cases lower than the first one, the oscillations are damped and represent regulating oscillations, which are typical for biological systems after perturbation.

Heat shock response of $A$. fumigatus characterized on the protein level

A sudden temperature change from $30^{\circ} \mathrm{C}$ to $48^{\circ} \mathrm{C}$ elicited a rapid alteration in protein expression and massive changes were already detectable after 30 minutes. The concurrent increase of HSPs of the cytoplasm, mitochondria and ER supports the general notion that heat shock is characterized by unfolding and disassembly of macromolecular structures, most notably proteins [50]. The relative abundance of most of the HSPs peaked at 120 minutes and only increased slightly thereafter or stayed at the same level. In this respect, the heat shock response of $A$. fumigatus does not differ significantly from that of yeast and other fungi. A transient increase of HSP transcripts within 60 minutes was also 
reported for S. cerevisiae and Schizosaccharomyces pombe. For these organisms, the maximum expression of HSP genes was detected at around 15 minutes $[11,12,51]$. HSP $30 /$ HSP42 and HSP 90 showed the highest increase in abundance during the heat shock response of A. fumigatus. HSP90 is a highly abundant cytosolic chaperone. It is essential for the proper function of a diverse set of key regulators of growth, development and defense and is known to be induced upon heat shock [52]. It was also described as an allergenic protein of A. fumigatus [53]. The alpha-crystalline-type HSP30 protein is highly upregulated in many filamentous fungi upon heat shock $[34,35,54]$. It is presumably involved in the import of proteins to the mitochondrion [55] and was shown to be upregulated under oxidative stress conditions in A. fumigatus [56].

Additionally, enzymes of the oxidative and nitrosative stress response were induced upon heat shock in $A$. fumigatus. It is known from yeast, that heat shock enhances oxygen respiration. This results in an increase in the formation of reactive oxygen intermediates (ROI) and an activation of the oxidative stress response [57]. The data indicate a putative heat shock-dependent upregulation of the thioredoxin peroxidase AspF3 and the cytochrome c peroxidase Ccp1. An upregulation of enzymes involved in the depletion of reactive nitrogen intermediates (RNI) upon heat shock has not been previously described for fungi. At higher temperature, higher amounts of reactive nitrogen intermediates may be endogenously generated, in particular during growth with nitrate as sole nitrogen source. Nitrate has to be assimilatory reduced to ammonium, a process during which RNI can be produced.

Interestingly, many differentially regulated proteins were involved in carbohydrate and nitrogen metabolism. Most of these enzymes showed an oscillatory upregulation with a maximum at 120 minutes. An increase in the abundance of proteins involved in glycolysis could provide energy needed for the ATP-dependent protein refolding by chaperones [11]. A heat shock-dependent regulation of the glycolytic enzymes phosphoglycerate kinase and hexokinase was also demonstrated for other fungi $[6,34,58]$.

In addition, enzymes of the NADPH-generating pentose phosphate pathway were upregulated. They are probably involved in balancing the redox state of $A$. fumigatus after heat stress by providing reducing equivalents for the reduction of ROI or oxidized glutathion or thioredoxin. The glucose-6-phosphate dehydrogenase was shown to be implicated in the adaptive response to oxidative stress in S. cerevisiae [59], but also showed a heat shock-dependent regulation in yeast [9]. Heat shock may also lead to increased fatty acid biosynthesis activity due to membrane lipid damage. The cytosolic
ATP-citrate lyase and the acetyl coenzyme A synthetase were upregulated after heat shock on the protein level [60]. These enzymes can provide acetyl-CoA as precursor for the biosynthesis of fatty acids.

Nitrogen metabolism was also affected by the temperature shift. Heat shock leads to an increased turnover of proteins and hence to a higher rate of amino acid biosynthesis in A. fumigatus. This is reflected by the increased levels of enzymes involved in the biosynthesis of amino acids. In contrast, amino acid synthesis is repressed in S. cerevisiae after heat shock [11,61]. Inconsistent to this regulation, many transcripts of amino acid biosynthesis genes showed a converse regulation in comparison to their corresponding protein in A. fumigatus. This phenomenon can best be explained by posttranscriptional regulation such as phosphorylation or acetylation.

Recently, a state space model was used to examine the regulation of heat shock and metabolism genes [18]. The study found a negative association of many HSPs and their regulated metabolic genes. It was most prominent in the temperature shift from $30^{\circ} \mathrm{C}$ to $37^{\circ} \mathrm{C}$ and much weaker at $48^{\circ} \mathrm{C}$. At the proteomic level, even this weak negative association cannot be confirmed, since all metabolic proteins showed a similar time course as the HSPs.

\section{Relation of transcriptome and proteome data}

Comparison of proteome and transcriptome data was not possible on a genome-wide scale. Only 32 proteins and respective transcripts were detected as differentially regulated in both datasets. Therefore, we analysed the correlation of each pair of transcript and protein separately, instead of looking for global relations. When the proteome data were compared with the transcript data of Nierman et al. [17], a low correlation for many transcripts and their corresponding proteins was found. Similar observations were made during a genome-wide analysis of the effect of heat shock on an S. cerevisiae mutant strain [33]. However, the transcript level of some genes, e.g. HSPs, correlated well with the corresponding protein levels. This was particularly true when a time delay between transcription and translation was taken into account. Part of this inconsistent regulation of transcripts and proteins is explainable by known regulation mechanisms. (i) In a wide variety of eukaryotes, many stress conditions including heat stress lead to a repression of translation initiation and an accumulation of translationally repressed mRNA in either so-called stress granules or in the nucleus. Under these conditions, mRNA is stabilized by the inhibition of poly(A) shortening, because deadenylation normally leads to an increased mRNA decay [62]. (ii) Under conditions of heat shock, mRNAs of HSP genes are selectively exported from the nucleus for protein biosynthesis, 
whereas the bulky poly(A) mRNA remains in the nucleus [63]. However, it cannot be ruled out that the different $A$. fumigatus strains and media used by us (AMM) and Nierman et al (complete media) caused a difference in gene and protein expression.

For the comparison of proteomic and transcriptomic data, two correlation measures were used. Both are qualitative measures. They result in only one value for each transcript and protein pair. In the future, it would also be interesting to use methods which provide quantitative information for the comparison of data from both cellular levels.

Putative Hsf binding sequences in the promoter region of heat-shock regulated proteins

The target genes of the transcriptional regulator Hsf1 contain a cis-acting sequence, the heat shock element (HSE). Using ScanProSite, 24 HSEs in promoter regions of 17 putative target genes of Hsf1 were identified in $A$. fumigatus. Using MEME, 5 additional motifs and 2 further putative target genes were identified. They had at most one modification that has not been reported in the literature. All three known HSE motifs (perfect, gap and step type, see additional file 4 for motif consensus sequences) were present in the promoter region of heatshock induced genes. Among the putatively Hsf-regulated genes (Figure 5), many known Hsf-targets were found such as HSPs, a thioredoxin peroxidase (AspF3), enzymes of the pentosephosphate shunt (6-phosphogluconate dehydrogenase) and glycolysis (hexokinase) $[9,58]$. However, other $A$. fumigatus genes with an HSEelement have so far not been associated with Hsf-regulation. In yeast, the cytochrome c peroxidase was shown to be regulated by the Msn2/Msn4-dependent general stress response [7], but in A. fumigatus its expression is presumably induced by Hsf1. The cytochrome c peroxidase degrades ROI in mitochondria and is involved in conveying the oxidative stress signal [64].

Hsf1 may also be a regulator of tRNA/rRNA transcription (protein phosphatase 2a, histidyl-tRNA synthetase, glycine-rich RNA binding protein), translation (eukaryotic translation initiation factor 4) and leucine biosynthesis (3-isopropylmalate dehydratase). Interestingly, the mannitol-1-phosphate dehydrogenase is also a potential target of Hsf1 in A. fumigatus. It is the first enzyme in the pathway for the biosynthesis of mannitol, which appears to be essential for the protection against various stress conditions including heat as shown in $A$. niger [65].

The protein NudC is involved in nuclear migration during mitosis and hyphal tip growth in A. nidulans, but also implicated in fungal cell wall biosynthesis [66]. Under heat stress conditions, the transcription of the NudC gene may be under transcriptional control of Hsf1 to maintain cytokinesis and cell proliferation at higher temperature. Interestingly, the NudC orthologue NUD-1 in the nematode Caenorhabditis elegans exhibits chaperone activity [67].

Of the aforementioned putatively Hsf1 regulated genes, mannitol-1-phosphate $\mathrm{DH}$, cytochrome c peroxidase and NudC showed the most typical Hsf1-driven rapid upregulation on the transcript level and a corresponding increase of the protein product (Figure 5). Interestingly, in the promoter region of the orthologous genes in A. flavus, A. nidulans and A. terreus, an HSEsequence was not detectable. Further studies are needed to verify whether this regulation is unique to A. fumigatus and whether it could contribute to the observed thermotolerance in this fungus.

Furthermore, the relevance of the AP- $2 \alpha$-like binding motif in the promoter region of some heat-induced genes has to be validated experimentally. Homologues of AP-2 like transcription factors have not been found in fungal genomes yet.

\section{Conclusions}

In our proteome study, the heat shock response of $A$. fumigatus was analyzed. For this purpose, a workflow was established that includes missing value imputation, data normalization and filtering of differentially regulated proteins. The workflow is shown in additional file 5 (workflow.tif). This workflow could be applicable to many DIGE datasets. Therefore, the described tool is publicly available in the internet.

The analysis of the adaptation of $A$. fumigatus to high temperatures revealed many similarities but also some obvious differences to the heat shock response compared to well-studied, mesophilic fungi such as S. cerevisiae and $S$. pombe, which are not able to grow at temperatures beyond $40^{\circ} \mathrm{C}$.

The heat shock response in A. fumigatus was transient and most changes in protein expression appeared within two hours. Afterwards, the level of protein expression continued on a different, often higher level (e. g. HSPs) or dropped to the initial level (e.g. glycolytic enzymes). Besides the well known increased biosynthesis of HSPs upon heat stress, several other processes were influenced in A. fumigatus during heat shock: oxidative stress response, signal transduction, transcription, translation, energy generation, carbohydrate and nitrogen metabolism and cytoskeleton organisation. Additionally to the Hsf1 regulon, other stress regulators are most likely involved in the heat shock response of $A$. fumigatus.

Novel potential Hsf1 targets were identified. They seem to be $A$. fumigatus specific and mediate oxidative stress resistance in mitochondria, function as osmoregulator or ROI scavenger or are required for nuclear migration. 
It is interesting to speculate that some of the putatively Hsf1-regulated target proteins described here mediate the high thermotolerance of $A$. fumigatus.

\section{Methods}

\section{Experimental Design}

In this study, the fungal response to heat shock at the time points $0,30,60,120$, and 180 minutes after a shift from $30^{\circ} \mathrm{C}$ to $48^{\circ} \mathrm{C}$ was analyzed. These time points were chosen to enable a good comparison with recent transcriptomic temperature shift data from $A$. fumigatus [17]. The temperature of $48^{\circ} \mathrm{C}$ was chosen to induce a clear heat shock response with an induction of heat shock responsive genes in the thermotolerant fungus [17].

An extended loop design [26] was applied, using two biological replicates which included four technical replicates in dye swap (Figure 1D). This design allows creating eight replicates for each of the five time points by using only 20 DIGE gels.

\section{Strains and culture condition}

Aspergillus fumigatus wild-type strain ATCC 46645 was cultivated in Aspergillus minimal medium (AMM) with glucose as sole carbon and energy source as described in Weidner et al. [68]. Flasks containing $100 \mathrm{ml}$ AMM were inoculated to a concentration of $3 \times 10^{6}$ conidia $/ \mathrm{ml}$ and incubated overnight on a rotary shaker with 200 $\mathrm{rpm}$ at $30^{\circ} \mathrm{C}$. After 16 hours, heat shock was induced by cultivating part of the cultures for four hours at $48^{\circ} \mathrm{C}$. Control cultures were kept at $30^{\circ} \mathrm{C}$. Mycelium was harvested by filtering the culture through Miracloth (Calbiochem, Germany). Subsequently, the mycelium was rinsed with demineralized water, pressed to remove liquid, frozen in liquid nitrogen, and stored at $-70^{\circ} \mathrm{C}$.

\section{DIGE 2D-gel electrophoresis}

Samples for two-dimensional polyacrylamide gel electrophoresis (2D-PAGE) were prepared as previously described [56,69]. Briefly, frozen mycelium was ground in a pre-cooled mortar with a pestle in the presence of liquid nitrogen. Proteins of the crude extract were precipitated by TCA/acetone as described in Kniemeyer et al. [69]. The air-dried pellet was dissolved in lysis buffer containing $7 \mathrm{M}$ urea, $2 \mathrm{M}$ thiourea, 2\% (w/v) CHAPS, $1 \%(\mathrm{w} / \mathrm{v})$ Zwittergent 3-10, and $30 \mathrm{mM}$ Tris. The $\mathrm{pH}$ of the sample was adjusted to 8.5 by addition of $100 \mathrm{mM}$ $\mathrm{NaOH}$. Protein concentration was determined according to the method of Bradford [70] using BIO-RAD protein assay (BIO-RAD Lab., Hartfordshire, USA). Afterwards, $50 \mu \mathrm{g}$ protein samples were labeled with $30 \mathrm{pmol}$ of CyDyes according to Lessing et al. [56]

Rehydrated IPGstrips (7 M urea, $2 \mathrm{M}$ thiourea, 2\% [w/ v] CHAPS, 1\% [w/v] Zwittergent 3-10, 0.002\% [w/v] bromophenol blue, $0.5 \%$ [vol/vol] IPG buffer 3-11, 1.2\% [vol/vol] De-Streak reagent) of $24 \mathrm{~cm}$ covering a nonlinear $\mathrm{pH}$ range from 3-11 were used for isoelectric focusing. $150 \mu \mathrm{g}$ protein of pooled, mixed samples and in addition $100 \mu \mathrm{g}$ unlabelled protein mixture were loaded via anodic cup loading onto IPGstrips. Isoelectric focusing was carried out as described previously [69]. Equilibrated IPGstrips were placed on $12.5 \%$ polyacrylamide gels, fixed with melted agarose and separated using an Ettan DALTsix electrophoresis system. Protein spots were visualized by a Typhoon 9410 scanner (GE Healthcare Biosciences) using a resolution of $100 \mu \mathrm{m}$. Images were cropped and spots were detected and quantified with the DeCyder 6.5 software package (GE Healthcare Biosciences). All proteins were subsequently visualized by Colloidal Coomassie staining as described by Neuhoff et al. [71].

\section{Protein identification by MS}

Significantly regulated proteins were excised and tryptically digested according to the protocol of Shevchenko et al [72]. Peptides were extracted for one hour with acetonitrile (ACN): trifluoracetic acid (TFA) $0.1 \%(1: 1 \mathrm{v} /$ $\mathrm{v})$, mixed with saturated a-cyano-4-hydroxycinnamic acid in ACN:TFA $0.1 \%(1: 2 \mathrm{v} / \mathrm{v})$ and allowed to dry on an MTP 800/384 anchor chip target (Bruker Daltonics, Germany). The samples were measured on an Ultraflex I MALDI-TOF/TOF device using flexControl 3.0 for data collection and flexAnalysis 3.0 (Bruker Daltonics $\mathrm{GmbH}$, Germany) for spectra analysis/peak list generation as described in Vödisch et al. [73]. Up to five peptides of the peptide mass fingerprint (PMF) spectra were chosen for post source decay MS/MS analyses. For identification, peptide mass fingerprint (PMF) and peptide fragmentation fingerprint (PFF) spectra were submitted to the MASCOT server (MASCOT 2.1.02, Matrix Science, UK), searching the taxon fungi in the NCBI database. With respect to the sample preparation, fixed modification of cysteins to S-carbamidomethyl derivatives and variable methionine oxidation was defined for the database search. Further, no missed cleavage, and a peptide mass tolerance of $50 \mathrm{ppm}$ was allowed. Results were regarded as significant with an allowed likelihood for a random hit of $\mathrm{p}=0.05$, according to the MASCOT score. Database searches were triggered and archived on a Proteinscape 1.3 database server (Protagen, Germany). Accuracy of raw peak lists was improved by automated internal recalibration using known contaminants (trypsin and keratin fragments) and application of the peak rejection filter of the Score Booster tool, implemented into the Proteinscape 1.3 database software.

\section{Image and data analysis}

Quantification of protein expression was carried out with DeCyder 6.5 and resulted in 1886 spots. Raw data were exported out of DeCyder and analysis was done using the statistical software $\mathrm{R}$ (version 2.8.0 using Bioconductor packages impute, vsn, limma and sma). 
In general, not all spots can be found on all gels by DeCyder and therefore show missing intensity values. In a first step, missing intensity values were imputed. Spots which were absent in six or seven of the eight replicates of one condition were considered as absent proteins in this condition (and thus, the one or two present signals as noise). They were imputed using a Gaussian distribution as described by Chich et al. [28] with the minimal detected value of all gels as mean $m$ and a variance of $s^{2}$ $=\mathrm{m} / 3$. This assures very low, but positive, intensity values. They are a little different from each other to preserve experimental variation for those spots. Spots which then had missing values in at most $25 \%$ of all gels (across all replicates) were imputed by k-nearest neighbor (knn) method as proposed by Jung et al [48]. All spots which still had missing values after this procedure were discarded, leaving 982 spots for analysis.

Second, data were normalized by variance stabilization (vsn) followed by local polynomial regression (loess) as described previously [31]. Additionally, after vsn and before loess, the internal Cy2 standard was used to remove gel specific differences. Subsequently, for each spot the median of intensities of all gels at the same time point was calculated. Median intensities of each time point were then divided by median intensity of time point $\mathrm{t}=0$ and logarithmized.

$\log _{2}$-ratios of normalized spot intensities were filtered for differential regulation as described before [31] using Z-Scores and ANOVA. Z-Scores for each spot were calculated for each time point separately. All spots with at least one Z-Score outside the range of $(-1.96,1.96)$, representing the $95 \%$ confidence level, were regarded as being differentially regulated. This resulted in spots with $\log _{2}$-ratios outside the range of $(-1.070,1.020)$, representing $(-2.099,2.028)$ as fold change thresholds. Additionally, ANOVA p-values (corrected for multiple testing by the method of Benjamini and Hochberg [74]) of smaller than 0.05 were used as indicators for statistical significance. Spots were regarded as being significantly upregulated when at least one $\log _{2}$-ratio (between one time point and time point 0 ) was above 1.020 , the maximal $\log _{2}$-ratio was larger than the absolute value of the minimal one (in case, a spot was first up- and then downregulated or vice versa) and when they showed an ANOVA p-value smaller than 0.05. Spots were regarded as being significantly downregulated when at least one $\log _{2}$-ratio was below -1.070 , the absolute value of the minimal $\log _{2}$-ratio was larger than the maximal one and when they showed an ANOVA p-value smaller than 0.05 .

Identified significant differentially regulated proteins were categorized according to the Functional Catalogue (FunCat [40]). FunCat information was taken from the PEDANT server of Munich Information Center for
Protein Sequences [75]. First, top level categories were analyzed to get a broad overview of the functional categories. Second, an enrichment analysis using Fisher's exact test with all known categories for the whole proteome as reference set was conducted.

\section{Comparison to transcriptomic data}

Pre-processed transcriptomic data, published in 2005 [17], were obtained from ArrayExpress [76] with accession number E-MEXP-332. For half of the differentially regulated proteins, the respective transcripts were also differentially regulated. Pearson (PC) and Spearman correlation (SC) for pairs of transcripts and respective proteins were calculated to depict relationships. Both were also calculated for time shifted proteomic data to find possible delays in translational regulation with respect to transcriptional regulation. Therefore, time points were interpolated every 15 minutes by means of adjacent time points.

\section{Comparison to yeast}

NCBI ProteinBlast was used to find homologues of the differentially regulated proteins of this study in S. cerevisiae. ScanProsite [45] was applied to search for the well known transcription factor binding sites (TFBSs) of Hsf1, Msn2/4 and Hac1 from $S$. cerevisiae $[11,12,14,43,44]$ in the differentially regulated proteins of A. fumigatus. Input were intergenic regions comprising up to $1000 \mathrm{bp}$ upstream of the transcript (less in case that another transcript was located less that $1000 \mathrm{bp}$ away from the analyzed one). Sequences were retrieved from CADRE [77] and adjusted. The proteins with detected TFBSs and the yeast homologues with known binding TFBSs were used as input in MEME [46] to find more proteins with little substitutions in the binding site. Additionally, promoters of all significant differentially regulated proteins were used to find additional, new motifs.

\footnotetext{
Additional file 1: MS_results. Results of the MALDI-TOF/TOF analysis of protein spots with significant change in abundance after heat shock. Click here for file

[http://www.biomedcentral.com/content/supplementary/1471-2164-1132-S1.XLS ]

Additional file 2: Funcat. FunCat annotations for all 64 differentially regulated proteins on most detailed (sheet1), second general (sheet2) and most general level (sheet4), including enrichment analysis of second level annotations (sheet3).

Click here for file

[http://www.biomedcentral.com/content/supplementary/1471-2164-1132-S2.XLS ]

Additional file 3: Time_series_tr_pr. Graphical display of the time series data of differentially expressed transcripts and proteins. Only significantly regulated proteins/transcripts are depicted. Time is given in minutes and the relative abundance of protein spots in $\log _{2}$ ratios. Each pair of a transcript and its respective protein is displayed in a separate plot. Transcripts are depicted in red, proteins in blue. If there were several spots representing the same protein, several blue lines were drawn.

Click here for file
} 
[http://www.biomedcentral.com/content/supplementary/1471-2164-1132-S3.DOC ]

Additional file 4: Sequence_analysis. Detected Hsf1 binding motifs in the upstream region of the genes representing the differentially regulated proteins (sheet1) and yeast homologues with their regulation (sheet2).

Click here for file

[http://www.biomedcentral.com/content/supplementary/1471-2164-1132-S4.XLS ]

Additional file 5: Workflow. The DIGE analysis workflow of this study. Click here for file

[http://www.biomedcentral.com/content/supplementary/1471-2164-1132-S5.TIFF]

\section{Acknowledgements}

Silke Steinbach is gratefully acknowledged for technical assistance. We thank Juliane Gebauer for calculating the correlations. We also thank Ekaterina Shelest for help with the sequence analysis as well as Olaf Scheibner and Robert Winkler for MS analysis. Additionally, Duncan Wilson is acknowledged for improving the English

This work was supported by the Deutsche Forschungsgemeinschaft (DFG) Priority Program 1160 'Colonisation and infection by human-pathogenic fungi' (to $A A B$ and $R G$ ).

\section{Author details}

'Research Group Systems Biology/Bioinformatics, Leibniz Institute for Natural Product Research and Infection Biology, Hans-Knöll-Institute, Jena, Germany. ${ }^{2}$ Department of Molecular and Applied Microbiology, Leibniz Institute for Natural Product Research and Infection Biology, Hans-Knöll-Institute, Jena, Germany. ${ }^{3}$ Friedrich Schiller University, Jena, Germany.

\section{Authors' contributions}

DA participated in the design of the study, carried out all bioinformatical analyses, and drafted the manuscript. RG and AAB initiated the study, participated in designing the experiments and writing the paper. OK carried out the experimental work, participated in the design of the study and took part in drafting the manuscript. All authors read and approved the final manuscript.

Received: 3 August 2009

Accepted: 15 January 2010 Published: 15 January 2010

\section{References}

1. Tekaia F, Latge JP: Aspergillus fumigatus: saprophyte or pathogen?. Curr Opin Microbiol 2005, 8:385-392.

2. Brakhage AA, Langfelder K: Menacing mold: the molecular biology of Aspergillus fumigatus. Annu Rev Microbiol 2002, 56:433-455.

3. Bhabhra R, Askew DS: Thermotolerance and virulence of Aspergillus fumigatus: role of the fungal nucleolus. Med Mycol 2005, 43(Suppl 1):S8793.

4. Zhou H, Hu H, Zhang L, Li R, Ouyang H, Ming J, Jin C: OMannosyltransferase 1 in Aspergillus fumigatus (AfPmt1p) is crucial for cell wall integrity and conidium morphology, especially at an elevated temperature. Eukaryot Cell 2007, 6:2260-2268.

5. Chang YC, Tsai HF, Karos M, Kwon-Chung KJ: THTA, a thermotolerance gene of Aspergillus fumigatus. Fungal Genet Biol 2004, 41:888-896.

6. Boucherie H, Sagliocco F, Joubert R, Maillet I, Labarre J, Perrot M: Twodimensional gel protein database of Saccharomyces cerevisiae. Electrophoresis 1996, 17:1683-1699.

7. Boy-Marcotte E, Lagniel G, Perrot M, Bussereau F, Boudsocq A, Jacquet M, Labarre J: The heat shock response in yeast: differential regulations and contributions of the Msn2p/Msn4p and Hsf1p regulons. Mol Microbiol 1999, 33:274-283.

8. Causton HC, Ren B, Koh SS, Harbison CT, Kanin E, Jennings EG, Lee TI, True HL, Lander ES, Young RA: Remodeling of yeast genome expression in response to environmental changes. Mol Biol Cell 2001, 12:323-337.
9. Eastmond DL, Nelson HC: Genome-wide analysis reveals new roles for the activation domains of the Saccharomyces cerevisiae heat shock transcription factor ( $\mathrm{Hsf} 1)$ during the transient heat shock response. J Biol Chem 2006, 281:32909-32921.

10. Garrels JI, McLaughlin CS, Warner JR, Futcher B, Latter Gl, Kobayashi R, Schwender B, Volpe T, Anderson DS, Mesquita-Fuentes R, Payne WE: Proteome studies of Saccharomyces cerevisiae: identification and characterization of abundant proteins. Electrophoresis 1997, 18:1347-1360

11. Gasch AP, Spellman PT, Kao CM, Carmel-Harel O, Eisen MB, Storz G, Botstein D, Brown PO: Genomic expression programs in the response of yeast cells to environmental changes. Mol Biol Cell 2000, 11:4241-4257.

12. Hahn JS, Hu ZZ, Thiele DJ, lyer VR: Genome-wide analysis of the biology of stress responses through heat shock transcription factor. Mol Cel Biol 2004, 24:5249-5256.

13. Estruch F: Stress-controlled transcription factors, stress-induced genes and stress tolerance in budding yeast. FEMS Microbiol Rev 2000, 24:469486.

14. Yamamoto A, Mizukami Y, Sakurai $\mathrm{H}$ : Identification of a novel class of target genes and a novel type of binding sequence of heat shock transcription factor in Saccharomyces cerevisiae. J Biol Chem 2005, 280:11911-11919.

15. Urano F, Bertolotti A, Ron D: IRE1 and efferent signaling from the endoplasmic reticulum. J Cell Sci 2000, 113(Pt 21):3697-3702.

16. Guthke R, Kniemeyer O, Albrecht D, Brakhage A, U M: Discovery of gene regulatory networks in Aspergillus fumigatus. Lect Notes Bioinf 2007, 4366:22-41.

17. Nierman WC, Pain A, Anderson MJ, Wortman JR, Kim HS, Arroyo J, Berriman M, Abe K, Archer DB, Bermejo C, et al: Genomic sequence of the pathogenic and allergenic filamentous fungus Aspergillus fumigatus. Nature 2005, 438:1151-1156.

18. Do JH, Yamaguchi R, Miyano S: Exploring temporal transcription regulation structure of Aspergillus fumigatus in heat shock by state space model. BMC Genomics 2009, 10:306.

19. Chang J, Van Remmen H, Ward WF, Regnier FE, Richardson A, Cornell J: Processing of data generated by 2-dimensional gel electrophoresis for statistical analysis: missing data, normalization, and statistics. J Proteome Res 2004, 3:1210-1218.

20. Fodor IK, Nelson DO, Alegria-Hartman M, Robbins K, Langlois RG, Turteltaub KW, Corzett TH, MCCutchen-Maloney SL: Statistical challenges in the analysis of two-dimensional difference gel electrophoresis experiments using DeCyder. Bioinformatics 2005, 21:3733-3740.

21. Kultima K, Scholz B, Alm H, Skold K, Svensson M, Crossman AR, Bezard E, Andren PE, Lonnstedt I: Normalization and expression changes in predefined sets of proteins using $2 \mathrm{D}$ gel electrophoresis: a proteomic study of L-DOPA induced dyskinesia in an animal model of Parkinson's disease using DIGE. BMC Bioinformatics 2006, 7:475.

22. Alban A, David SO, Bjorkesten L, Andersson C, Sloge E, Lewis S, Currie I: A novel experimental design for comparative two-dimensional gel analysis: two-dimensional difference gel electrophoresis incorporating a pooled internal standard. Proteomics 2003, 3:36-44.

23. Marouga R, David S, Hawkins E: The development of the DIGE system: 2D fluorescence difference gel analysis technology. Anal Bioanal Chem 2005, 382:669-678.

24. Tannu NS, Hemby SE: Two-dimensional fluorescence difference gel electrophoresis for comparative proteomics profiling. Nat Protoc 2006, 1:1732-1742.

25. Timms JF, Cramer R: Difference gel electrophoresis. Proteomics 2008, 8:4886-4897.

26. Altman NS, Hua J: Extending the loop design for two-channel microarray experiments. Genet Res 2006, 88:153-163.

27. Ahmad N, Zhang J, Brown PJ, James DC, Birch JR, Racher AJ, Smales CM: On the statistical analysis of the GS-NSO cell proteome: imputation, clustering and variability testing. Biochim Biophys Acta 2006, 1764:11791187.

28. Chich JF, David O, Villers F, Schaeffer B, Lutomski D, Huet S: Statistics for proteomics: experimental design and 2-DE differential analysis. J Chromatogr B Analyt Technol Biomed Life Sci 2007, 849:261-272.

29. Jung K, Gannoun A, Sitek B, Apostolov O, Schramm A, Meyer HE, Stühler K, Urfer W: Statistical evaluation of methods for the analysis of dynamic protein expression data from a tumor study. REVSTAT 2006, 4:67-80. 
30. Albrecht $\mathrm{D}$, Kniemeyer $\mathrm{O}$, Brakhage A, Guthke R: Missing values in gelbased proteomics. Proteomics 2010, 9(5):1407-15.

31. Albrecht $D$, Kniemeyer $O$, Brakhage AA, Guthke R: Normalisation of 2D DIGE Data - on the Way to a Standard Operating Procedure. BIRD'08 2nd International Conference on Bioinformatics Research and Development Schriftenreihe Informatik 26. Linz: Trauner VerlagKüng J, Schneider K, Wagner R 2008, 55-64.

32. Albrecht $D$, Kniemeyer $O$, Brakhage AA, Berth $M$, Guthke R: Integration of transcriptome and proteome data from human-pathogenic fungi by using a data warehouse. J Integrative Bioinf 2007, 4:52.

33. Jones DL, Petty J, Hoyle DC, Hayes A, Oliver SG, Riba-Garcia I, Gaskell SJ, Stateva $L$ : Genome-wide analysis of the effects of heat shock on a Saccharomyces cerevisiae mutant with a constitutively activated CAMPdependent pathway. Comp Funct Genomics 2004, 5:419-431.

34. Chandler JM, Treece ER, Trenary HR, Brenneman JL, Flickner TJ, Frommelt JL, Oo ZM, Patterson MM, Rundle WT, Valle OV, et al: Protein profiling of the dimorphic, pathogenic fungus, Penicillium marneffei. Proteome Sci 2008, 6:17.

35. Georgianna DR, Hawkridge AM, Muddiman DC, Payne GA: Temperaturedependent regulation of proteins in Aspergillus flavus: whole organism stable isotope labeling by amino acids. J Proteome Res 2008, 7:2973-2979.

36. Zhou S, Fushinobu S, Nakanishi Y, Kim SW, Wakagi T, Shoun H: Cloning and characterization of two flavohemoglobins from Aspergillus oryzae. Biochem Biophys Res Commun 2009, 381:7-11.

37. de Oliveira IM, Henriques JA, Bonatto D: In silico identification of a new group of specific bacterial and fungal nitroreductases-like proteins. Biochem Biophys Res Commun 2007, 355:919-925.

38. Kosmidou E, Lunness $\mathrm{P}$, Doonan $\mathrm{JH}$ : A type $2 \mathrm{~A}$ protein phosphatase gene from Aspergillus nidulans is involved in hyphal morphogenesis. Curr Genet 2001, 39:25-34

39. Oficjalska-Pham D, Harismendy O, Smagowicz WJ, Gonzalez de Peredo A, Boguta M, Sentenac A, Lefebvre O: General repression of RNA polymerase III transcription is triggered by protein phosphatase type 2A-mediated dephosphorylation of Maf1. Mol Cell 2006, 22:623-632.

40. Ruepp A, Zollner A, Maier D, Albermann K, Hani J, Mokrejs M, Tetko I, Guldener U, Mannhaupt G, Munsterkotter M, Mewes HW: The FunCat, a functional annotation scheme for systematic classification of proteins from whole genomes. Nucleic Acids Res 2004, 32:5539-5545http://mips.gsf. de/projects/funcat.

41. Greenbaum D, Colangelo C, Williams K, Gerstein M: Comparing protein abundance and mRNA expression levels on a genomic scale. Genome Biol 2003, 4:117.

42. Gygi SP, Rochon Y, Franza BR, Aebersold R: Correlation between protein and mRNA abundance in yeast. Mol Cell Biol 1999, 19:1720-1730.

43. Wu WS, Li WH: Identifying gene regulatory modules of heat shock response in yeast. BMC Genomics 2008, 9:15.

44. Yamamoto $\mathrm{N}$, Maeda $\mathrm{Y}$, Ikeda A, Sakurai $\mathrm{H}$ : Regulation of thermotolerance by stress-induced transcription factors in Saccharomyces cerevisiae. Eukaryot Cell 2008, 7:783-790.

45. de Castro E, Sigrist CJ, Gattiker A, Bulliard V, Langendijk-Genevaux PS, Gasteiger E, Bairoch A, Hulo N: ScanProsite: detection of PROSITE signature matches and ProRule-associated functional and structural residues in proteins. Nucleic Acids Res 2006, 34:W362-365http://www. expasy.ch/tools/scanprosite.

46. Bailey TL, Williams N, Misleh C, Li WW: MEME: discovering and analyzing DNA and protein sequence motifs. Nucleic Acids Res 2006, 34:W369373http://meme.sdsc.edu/meme/

47. Eckert D, Buhl S, Weber S, Jager R, Schorle H: The AP-2 family of transcription factors. Genome Biol 2005, 6:246.

48. Jung K, Gannoun A, Sitek B, Meyer HE, Stühler K, Urfer W: Analysis of dynamic protein expression data. REVSTAT 2005, 3:99-111.

49. Kreil DP, Karp NA, Lilley KS: DNA microarray normalization methods can remove bias from differential protein expression analysis of $2 \mathrm{D}$ difference gel electrophoresis results. Bioinformatics 2004, 20:2026-2034.

50. Burnie JP, Carter TL, Hodgetts SJ, Matthews RC: Fungal heat-shock proteins in human disease. FEMS Microbiol Rev 2006, 30:53-88.

51. Chen D, Toone WM, Mata J, Lyne R, Burns G, Kivinen K, Brazma A, Jones N, Bahler J: Global transcriptional responses of fission yeast to environmental stress. Mol Biol Cell 2003, 14:214-229.

52. Picard D: Heat-shock protein 90 , a chaperone for folding and regulation. Cell Mol Life Sci 2002, 59:1640-1648.
53. Bowyer P, Denning DW: Genomic analysis of allergen genes in Aspergillus spp: the relevance of genomics to everyday research. Med Mycol 2007, 45:17-26.

54. Noguchi R, Banno S, Ichikawa R, Fukumori F, Ichiishi A, Kimura M, Yamaguchi I, Fujimura M: Identification of OS-2 MAP kinase-dependent genes induced in response to osmotic stress, antifungal agent fludioxonil, and heat shock in Neurospora crassa. Fungal Genet Biol 2007, 44:208-218.

55. Plesofsky N, Gardner N, Lill R, Brambl R: Disruption of the gene for Hsp30, an alpha-crystallin-related heat shock protein of Neurospora crassa, causes defects in import of proteins into mitochondria. Biol Chem 1999, 380:1231-1236.

56. Lessing F, Kniemeyer O, Wozniok I, Loeffler J, Kurzai O, Haertl A, Brakhage AA: The Aspergillus fumigatus transcriptional regulator AfYap1 represents the major regulator for defense against reactive oxygen intermediates but is dispensable for pathogenicity in an intranasal mouse infection model. Eukaryot Cell 2007, 6:2290-2302.

57. Sugiyama K, Izawa $S$, Inoue $Y$ : The Yap1p-dependent induction of glutathione synthesis in heat shock response of Saccharomyces cerevisiae. J Biol Chem 2000, 275:15535-15540.

58. Sakurai H, Takemori Y: Interaction between heat shock transcription factors (HSFs) and divergent binding sequences: binding specificities of yeast HSFs and human HSF1. J Biol Chem 2007, 282:13334-13341.

59. Izawa S, Maeda K, Miki T, Mano J, Inoue Y, Kimura A: Importance of glucose-6-phosphate dehydrogenase in the adaptive response to hydrogen peroxide in Saccharomyces cerevisiae. Biochem J 1998, 330(Pt 2):811-817.

60. Steels EL, Learmonth RP, Watson K: Stress tolerance and membrane lipid unsaturation in Saccharomyces cerevisiae grown aerobically or anaerobically. Microbiology 1994, 140(Pt 3):569-576.

61. Ye Y, Zhu Y, Pan L, Li L, Wang X, Lin Y: Gaining insight into the response logic of Saccharomyces cerevisiae to heat shock by combining expression profiles with metabolic pathways. Biochem Biophys Res Commun 2009, 385:357-362.

62. Hilgers $V$, Teixeira D, Parker R: Translation-independent inhibition of mRNA deadenylation during stress in Saccharomyces cerevisiae. RNA 2006, 12:1835-1845.

63. Izawa S, Kita T, Ikeda K, Inoue Y: Heat shock and ethanol stress provoke distinctly different responses in 3'-processing and nuclear export of HSP mRNA in Saccharomyces cerevisiae. Biochem J 2008, 414:111-119.

64. Charizanis C, Juhnke H, Krems B, Entian KD: The mitochondrial cytochrome c peroxidase $\mathrm{Ccp} 1$ of Saccharomyces cerevisiae is involved in conveying an oxidative stress signal to the transcription factor Pos9 (Skn7). Mol Gen Genet 1999, 262:437-447.

65. Ruiiter GJ, Bax M, Patel H, Flitter SJ, Vondervoort van de PJ, de Vries RP, vanKuyk PA, Visser J: Mannitol is required for stress tolerance in Aspergillus niger conidiospores. Eukaryot Ccell 2003, 2:690-698.

66. Chiu YH, Xiang X, Dawe AL, Morris NR: Deletion of nudC, a nuclear migration gene of Aspergillus nidulans, causes morphological and cell wall abnormalities and is lethal. Mol Biol Cell 1997, 8:1735-1749.

67. Faircloth LM, Churchill PF, Caldwell GA, Caldwell KA: The microtubuleassociated protein, NUD-1, exhibits chaperone activity in vitro. Cell Stress Chaperones 2009, 14:95-103.

68. Weidner G, d'Enfert C, Koch A, Mol PC, Brakhage AA: Development of a homologous transformation system for the human pathogenic fungus Aspergillus fumigatus based on the pyrG gene encoding orotidine 5'monophosphate decarboxylase. Curr Genet 1998, 33:378-385.

69. Kniemeyer O, Lessing F, Scheibner O, Hertweck C, Brakhage AA: Optimisation of a 2-D gel electrophoresis protocol for the humanpathogenic fungus Aspergillus fumigatus. Curr Genet 2006, 49:178-189.

70. Bradford MM: A rapid and sensitive method for the quantitation of microgram quantities of protein utilizing the principle of protein-dye binding. Anal Biochem 1976, 72:248-254

71. Neuhoff V, Arold N, Taube D, Ehrhardt W: Improved staining of proteins in polyacrylamide gels including isoelectric focusing gels with clear background at nanogram sensitivity using Coomassie Brilliant Blue G250 and R-250. Electrophoresis 1988, 9:255-262.

72. Shevchenko A, Jensen ON, Podtelejnikov AV, Sagliocco F, Wilm M, Vorm O, Mortensen P, Shevchenko A, Boucherie H, Mann M: Linking genome and proteome by mass spectrometry: large-scale identification of yeast 
proteins from two dimensional gels. Proc Natl Acad Sci 1996, 93:1444014445.

73. Vödisch M, Albrecht D, Lessing F, Schmidt AD, Winkler R, Guthke R, Brakhage AA, Kniemeyer O: Two-dimensional proteome reference maps for the human pathogenic filamentous fungus Aspergillus fumigatus. Proteomics 2009, 9:1407-1415.

74. Benjamini $Y$, Hochberg $Y$ : Controlling the false discovery rate: a practical and powerful approach to multiple testing. J R Stat Soc Ser B 1995, 57:289-300.

75. Frishman D, Albermann K, Hani J, Heumann K, Metanomski A, Zollner A, Mewes HW: Functional and structural genomics using PEDANT. Bioinformatics 2001, 17:44-57http://pedant.gsf.de/pedant3htmlview/ pedant3view?Method=analysis\&Db=p3_p131_Asp_fumig

76. Parkinson H, Kapushesky M, Shojatalab M, Abeygunawardena N, Coulson R, Farne A, Holloway E, Kolesnykov N, Lilja P, Lukk M, et al: ArrayExpress-a public database of microarray experiments and gene expression profiles. Nucleic Acids Res 2007, 35:D747-750http://www.ebi.ac.uk/microarray-as/ae.

77. Mabey JE, Anderson MJ, Giles PF, Miller CJ, Attwood TK, Paton NW, Bornberg-Bauer E, Robson GD, Oliver SG, Denning DW: CADRE: the Central Aspergillus Data REpository. Nucleic Acids Res 2004, 32:D401-405http:// www.cadre-genomes.org.uk/Aspergillus_fumigatus.

doi:10.1186/1471-2164-11-32

Cite this article as: Albrecht et al:: Integrative analysis of the heat shock response in Aspergillus fumigatus. BMC Genomics 2010 11:32.

Publish with Biomed Central and every scientist can read your work free of charge

"BioMed Central will be the most significant development for disseminating the results of biomedical research in our lifetime. "

Sir Paul Nurse, Cancer Research UK

Your research papers will be:

- available free of charge to the entire biomedical community

- peer reviewed and published immediately upon acceptance

- cited in PubMed and archived on PubMed Central

- yours - you keep the copyright 